\title{
A POTPOURRI OF IMPEDANCE MEASUREMENTS AT THE ADVANCED PHOTON SOURCE STORAGE RING CONF-970503--
}

\author{
N. S. Sereno, Y.-C. Chae, K. C. Harkay, A. H. Lumpkin, S. V. Milton B. X. Yang \\ Advanced Photon Source, Argonne National Laboratory \\ 9700 South Cass Avenue. Argonne, Illinois 60439 USA
}

\section{Abstract}

Machine coupling impedances were determined in the APS storage ring from measurements of the bunch length, synchronous phase. and synchrotron and betatron tunes vs single-bunch current. The transverse measurements were performed for various numbers of small gap insertion device (ID) chambers installed in the ring. The transverse impedance is determined from measurements of the transverse tunes and bunch length as a function of single-bunch current. The shift in the synchrotron tune was measured as a function of bunch current from which the total cavity impedance was extracted. The loss factor was determined by measuring the relative synchronous phase as a function of bunch current. The longitudinal resistive impedance is calculated using the loss factor dependence on the bunch length. From these results, we can estimate what the impedance would be for a full set of ID chambers.

\section{LONGITLDINAL}

The longitudinal coupling impedance, $Z_{\|}(\omega)$, is responsible for a number of effects which potentially degrade the performance of an accelerator or storage ring. Discussion will be limited to single-bunch effects; these include bunch lengthening, microwave instabilities, and chamber heating, all of which can reduce the beam lifetime. In the 7-GeV APS positron storage ring, the main contributions to $Z_{i l}$ are the if cavity higher-order modes (HOMs) and the broadband impedance due to vacuum chamber transitions, flanges, bellows, etc. A convenient quantity used as a measure of the total resistive impedance integrated over all frequencies is the loss factor, $k$. Loss factors may be associated with individual vacuum chamber components or with the entire accelerator as a whole. The reactive impedance. which changes the if focusing and shifts the synchrotron frequency. will not be discussed.

One method of measuring the loss factor is to observe the shift in the synchronous phase of a bunch [1]. The resistive impedance causes the beam to lose energy. which must be compensated by shifting the bunch to a phase corresponding to a higher field in the rf cavities. The shift in synchronous phase of an intense test bunch was measured relative to a small bunch used as a time reference. The reference bunch phase is solely determined by the currentindependent losses due to synchrotron radiation. $U_{\text {rad }}$, nominally $5.45 \mathrm{MeV} / \mathrm{tum}$. The current of the reference bunch was $0.6 \mathrm{~mA}$ and spaced halfway around the ring at $1.8 \mu$ s relative to the test bunch. while the test bunch current was varied from 1.3 to $6.4 \mathrm{~mA}$ at a tixed rf voltage of 9 MV. A Hamamatsu C $\$ 680$ dual-sweep streak camera was used to image the bunches using the optical synchrotron radiation from a bending magnet source. For each current value, 12 turns of data were acquired. The streak image intensity profiles for each bunch were averaged over the entire record, from which the centroids were computed.

The normalized intensity profiles for the test bunch are shown in Fig. 1. The time offset is measured relative to the reference bunch. As the bunch intensity increases, the energy loss increases, and the bunch centroid is observed to shift towards the head of the bunch (left in the figure). The centroid positions of each test bunch relative to the reference bunch are shown in Fig. 2. The shift is nearly linear with current. The energy loss is given by [1]

$$
\Delta \mathrm{U}=\mathrm{qV}_{\mathrm{rf}}\left|\sin \phi_{\mathrm{s}}-\sin \phi_{\mathrm{s} O}\right| \text {, }
$$

where $q$ is the bunch charge, $V_{r f}$ is the total rf voltage, $\phi_{s 0}$ is the zero-current synchronous phase angle, and $\phi_{\mathrm{S}}$ is the shifted phase. The loss factor, $\mathrm{k}_{\mathrm{TOT}}$, is defined as

$$
k_{\text {TOT }}=\frac{\Delta U}{q^{2}}=\frac{1}{2 \pi} \int_{-\infty}^{\infty} Z(\omega) \mathrm{e}^{-\omega^{2} \sigma_{\tau}^{2}} \mathrm{~d} \omega,
$$

where $I_{0}$ is the average bunch current, and a gaussian charge density with rms bunch length $\sigma_{\tau}$ is assumed.

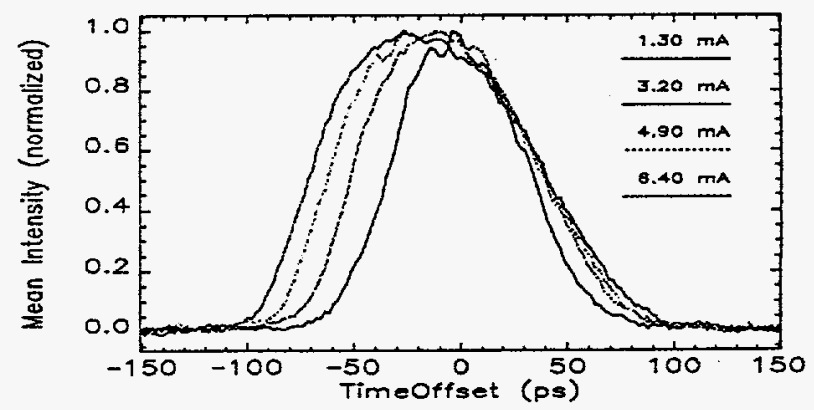

Figure 1: Normalized streak camera profiles for increasing test bunch current.

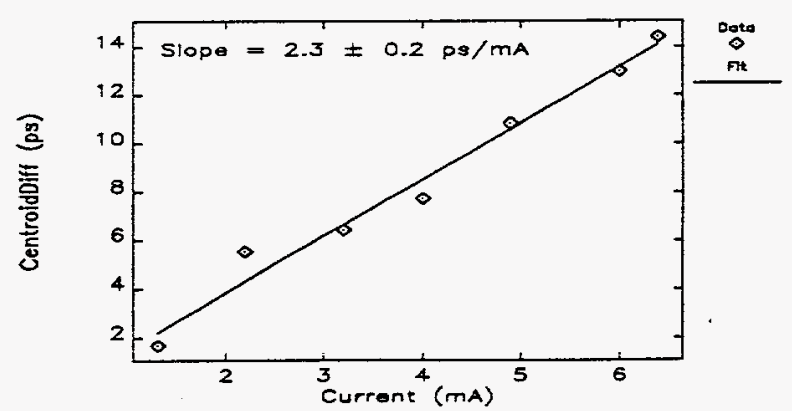

Figure 2: Centroid position shift of test bunch relative to reference bunch plotted vs. test bunch current.

The submitted manuscript has been created by the University of Chicago as Operator of Argonne National Laboratory ("Argonne") under Contract No. W-31-109-ENG-38 with the U.S. Department of Energy. The U.S. Government retains for itself, and others acting on its behalf, a paid-up, nonexclusive, irsevocable worldwide license in said article to reproduce, prepare derivative works. distribute copies to the public, and perform publicly and display publicly, by or on behalf of the Government. 


\section{DISCLAMMER}

Portions of this docoment may be illegible in electronic image products. Images are produced from the best available original docoment. 


\section{DISCLAIMER}

This report was prepared as an account of work sponsored by an agency of the United States Government. Neither the United States Government nor any agency thereof, nor any of their employees, make any warranty, express or implied, or assumes any legal liability or responsibility for the accuracy, completeness, or usefulness of any information, apparatus, product, or process disclosed, or represents that its use would not infringe privately owned rights. Reference herein to any specific commercial product, process, or service by trade name, trademark, manufacturer, or otherwise does not necessarily constitute or imply its endorsement, recommendation, or favoring by the United States Government or any agency thereof. The views and opinions of authors expressed herein do not necessarily state or reflect those of the United States Government or any agency thereof. 
Using Eqs. (1) and (2). and assuming a small shift. $\Delta \phi_{s}=\phi_{s}-\phi_{s 0}$, the loss tactor is computed using

$$
\mathrm{k}_{\mathrm{TOT}}=\operatorname{sign}(\eta) \mathrm{V}_{\mathrm{rf}} \cos \mathrm{O}_{\mathrm{s} O}\left(\frac{\Delta \phi_{\mathrm{s}}}{\mathrm{q}}\right)=10 \mathrm{~V} / \mathrm{pC},
$$

where $\eta$ is the slip factor and $\mathrm{L}_{\mathrm{rad}}=\mathrm{V}_{\mathrm{rf}} \sin \phi_{\mathrm{s} 0}$. The slope in Fig. 2 is $1.4 \mu \mathrm{rad} / \mathrm{pC}$ and is derived using $2.84 \mathrm{nsec}$ per if wave and a revolution frequency of $271.55 \mathrm{kHz}$. This value of $k_{\text {TOT }}$ may be compared to the loss factor estimated from the of cavity higher-order modes (HOMs). The program $\mathrm{ABCI}$ [2] was used to estimate the loss factor per cavity. The loss calculated was $0.43 \mathrm{~V} / \mathrm{pC}$ for an $\mathrm{rms}$ bunch length of $1 \mathrm{~cm}$. For 16 cavities in the ring, the HOMs contribute about $70 \%$ of the total intensity dependent energy loss while about $30 \%$ can be attributed to other sources. The loss factor dependence on bunch length could not be determined because of the small bunch length variation over these data.

A measurement of the local loss factor around the ring was attempted by measuring the position offset of a BPM in the high dispersion $(\eta=0.4 \mathrm{~m})$ positions in the ring. The measurement did not yield any systematic energy shift for single-bunch currents up to $5 \mathrm{~mA}$. There are two reasons for this. First the magnitude of the effect is very small; at $5 \mathrm{~mA}$, the expected position shift is only $10 \mu \mathrm{m}$ assuming all the loss $(10 \mathrm{~V} / \mathrm{pC})$ is located near a single high dispersion point. In reality, the loss is distributed around the ring so the position offsets expected are at least an order of magnitude less. Second and most important, the BPM offset intensity dependence is anywhere from 10 to $50 \mu \mathrm{m}$ as the current is varied by $5 \mathrm{~mA}$ [3]. At this time, there is no independent way to measure this dependence with current so that it may be separated out from the losses due to the local impedance.

\section{TRANSVERSE}

The transverse impedance $Z_{\perp}(\omega)$ describes the influence of various beamline components on the transverse dynamics of the beam. An important consideration for the APS storage ring is the transverse impedance due to a series of small-gap (8-mm vertical aperture) insertion device (ID) chambers that the beam must traverse. The wakefields generated in each chamber are relatively short, and hence it is the broadband impedance that will increase as more chambers are installed in the ring. The objective is to obtain an experimental value for the impedance of a single small-gap chamber to compare with the expected ring impedance.

The impedance generates coherent oscillation modes of the particles within a bunch or coupled-bunch modes of several bunches. For the measurements described here, a single bunch was used so that modes of oscillation of the particles in the single bunch are considered. The complex frequency shift for mode $m$ is given in terms of the effective transverse impedance by [ 4$]$

$$
\begin{gathered}
\frac{\delta \omega_{\mathrm{m}}}{\omega_{0}}=\mathrm{j}\left(\frac{\mathrm{I}}{\sigma}\right)\left(\frac{1 /(+\sqrt{\pi})}{1+\mathrm{m}}\right)\left(\frac{\bar{\beta} \mathrm{R}}{\mathrm{E} / \mathrm{e}}\right) \overline{\mathrm{Z}}_{\perp}(\omega), \\
\overline{\mathrm{Z}}_{\perp}(\omega) \equiv \frac{\sum \mathrm{Z}_{\perp}(\omega) \mathrm{h}_{\mathrm{m}}\left(\omega-\omega_{\xi}\right)}{\sum \mathrm{h}_{\mathrm{m}}\left(\omega-\omega_{\bar{\xi}}\right)},
\end{gathered}
$$

where $\bar{\beta}$ is the average ring beta function, $R$ is the ring radius, $I$ is the average beam current, $\sigma$ is the rms bunch length, $\mathrm{E} / \mathrm{e}$ is the energy expressed as a voltage, $\omega_{0}$ is the angular revolution frequency, $h_{m}\left(\omega-\omega_{\xi}\right)$ is the singlebunch spectrum shifted by frequency $\omega_{\xi}$, which depends on the chromaticity, and $\bar{Z}_{\perp}(\omega)$ is the effective transverse impedance due to all the ring components. The effective impedance is seen to be a convolution with the bunch power spectrum. Assuming the bunch spectrum is narrow, as is the case with long bunches in a proton ring, the impedance $Z_{\perp}(\omega)$ can be removed from the sum, and by varying the chromaticity the impedance can be determined as a function of frequency. This assumption is not valid for the short positron bunches in the APS ring which result in an rms spectral width $(-10 \mathrm{GHz})$ on the same order as $\omega_{\xi}$. Therefore it is only the effective transverse impedance that is obtained in this measurement.

The imaginary part of Eq. (3) gives the inverse growth rate of the particular mode $m$. The real part yields the frequency shift of mode $\mathrm{m}$. The mode $\mathrm{m}=0$ is the mode that is most strongly driven by the impedance and represents the rigid oscillation of all particles within the bunch. As the bunch current is increased, this mode is detected by observing the tune shift. Referring to Eq. (3), the imaginary part of the effective transverse impedance can be deduced from a measurement of the tune shift. The tunes were observed by driving a pair of striplines using a network analyzer and detecting beam motion using a similar pair of striplines [5].

In addition to the tunes, the bunch length is measured using the streak camera. The rms bunch length in the APS ring is observed to increase on the order of $50 \mathrm{ps}$ as the single-bunch current is varied on the order of $10 \mathrm{~mA}$ consistent with potential well distortion $[1,6]$. The bunch length vs current data are well fit by a 3rd-order polynomial as predicted by the potential well model. This fit is interpolated and used to compute the quantity $\mathrm{I} / \sigma$ in Eq. (3).

Figures 3 and 4 show the effective impedance for the horizontal and vertical planes deduced from three measurements for different numbers of small gap chambers installed in the ring. The horizontal axis of the figures are in units of the the standard 8-mm gap, 5-m long chamber used for IDs in the APS ring. The relatively large error bars in both figures reflect the tune measurement uncertainty which is due to the slope error of the linear fit to the tune vs $I / \sigma$ data. The effective impedance per chamber is derived from the slope of a linear fit to the three impedance values in each figure.

The effective horizontal transverse impedance per chamber is seen to have a very large uncertainty. This is 
due to the fact that the horizontal tune shift was observed * - to be very small and difficult to measure as compared to the vertical tune shift vs $I / \sigma$. The horizontal data are consistent with the fact that the chamber is very wide horizontally as compared to the vertical dimension, and therefore one expects the horizontal impedance to be smail.

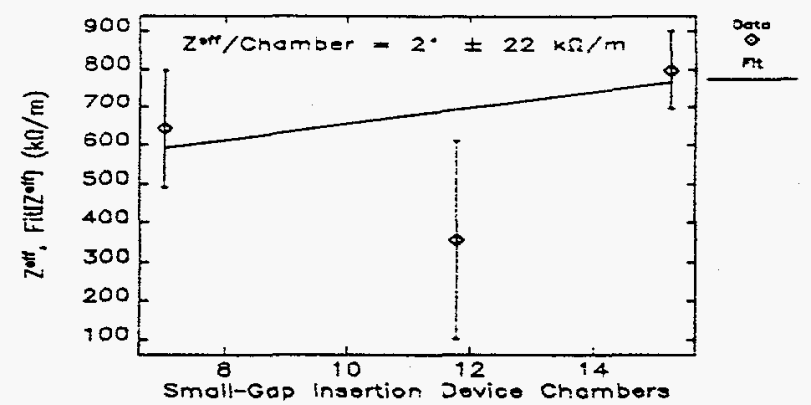

Figure 3: Horizontal transverse impedance as a function of the number of small-gap chambers in the ring.

The vertical data shown in figure 4 indicate a definite trend toward higher effective impedance as the number of chambers increases. The slope of the line indicates an effective transverse impedance of $53 \mathrm{k} \Omega / \mathrm{m}$ per chamber which is much larger than the anticipated value of 1.76 $\mathrm{k} \Omega / \mathrm{m}[7]$. The effective impedance is larger in part because it includes the resistive wall impedance. The anticipated value is computed neglecting the resistive wall part of the impedance. The large value is also due to the fact that the bunch spectrum for APS bunches is very wide and therefore the large broadband impedance at near $\omega=0$ contributes substantially to the sum in Eq. (2). Using the computed value of the effective vertical impedance per chamber, one would expect $1.8 \mathrm{M} \Omega / \mathrm{m}$ for the full complement of 34 chambers in the ring.

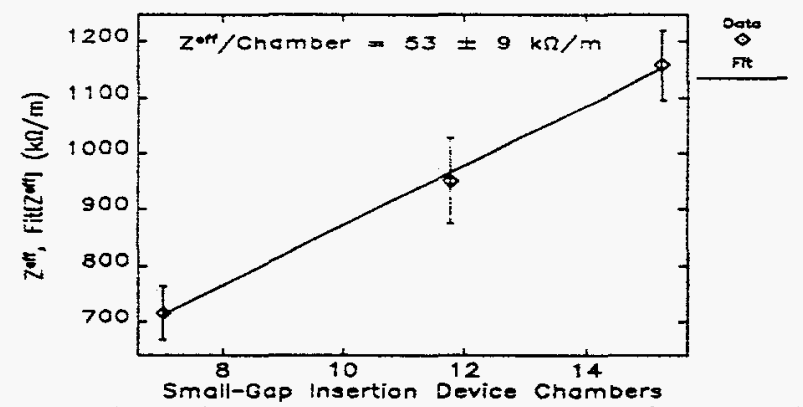

Figure 4: Vertical transverse impedance as a function of the number of small-gap chambers in the ring.

The local reactive component of the vacuum chamber transverse impedance was measured using a method first developed at CERN [8]. The BPM beam history modules were used to acquire vertical position data over 128 tums after the beam had been excited in the vertical plane using a kicker. This data was subsequently processed to determine the vertical betatron phase advance variation per $\mathrm{mA}$ as a function of position around the machine. Four positions in the machine were measured in this initial study.

The sum of the phase advance converted to tune units for all four positions results in $-4.6 / \mathrm{A}$. This value was found to be in agreement with measurements of the tune shift with current using the standard tune measurement technique [4]. The measured values reveal qualitatively that the region with the $\mathrm{rf}$. injection elements, and ID chambers have a larger effect on the beam (larger tune shift with current) than a region with standard vacuum chamber components. More measurements will be made to determine in detail the transverse impedance around the ring at more than the four points done in this exploratory measurement.

\section{CONCLUSIONS}

The longitudinal loss factor was determined by measurement of the synchronous phase vs single-bunch current for the APS ring. The loss due to HOMs in the cavities was estimated by a calculation so that losses due to other sources around the ring could be estimated. An attempt was made to measure the local loss around the ring at high dispersion areas. This proved to be difficult primarily due to the relatively large BPM offset intensity dependence. The effective transverse impedance was determined for the 8-mm small-gap ID chambers by measuring the tune shift as a function of single-bunch current. The result was a large vertical transverse impedance relative to horizontal due to the small vertical aperture of the chambers. A measurement was made using the beam history modules to estimate the local effective transverse impedance around the ring. The data confirm qualitatively the high transverse impedance features of the APS ring.

\section{ACKNOWLEDGEMENTS}

The authors would like to thank M. Borland, G. Decker, L. Emery, and J. Galayda for useful comments and suggestions regarding this work. This work was supported by U.S. Department of Energy, Office of Basic Energy Sciences, under Contract No. W-31-109-ENG-38.

\section{REFERENCES}

[1] H. Wiedemann. Particle Accelerator Physics 11, Springer-Verlag 207 (1995).

[2] Y. H. Chin, "Users Guide for New ABCI Version 6.1," CERN SL/ 92-49 (1992).

[3] L. Emery, M. Borland "Advances in Orbit Drift Correction in the Advanced Photon Source Storage Ring," these proceedings.

[4] F. Sacherer, B. Zotter, "Transverse Instabilities of Relativistic Particle Beams in Accelerators and Storage Rings," CERN 77-13 (1977).

[5] W. Sellyey, E. Kahana, and X. Wang. "Tune Measurement in the APS Rings." Proceedings of the 1993 Particle Accelerator Conference. Washington DC. 2237 (1993).

[6] A. H. Lumpkin, B. X. Yang, Y. C. Chae, "Observations of BunchLengthening Effects in the APS 7-GeV Storage Ring," Proceedings of 1996 FEL Conf.. NIM-A (1997) (in print).

[7] L. Emery, private communication.

[8] D. Brandt et al. "Measurements of Impedance Distributions and Instability Thresholds in LEP." Proceedings of the 1995 Particle Accelerator Conference. Dallas. TX. 570 (1995). 